\title{
Comment on Minimally Invasive Surgical Approach for Treatment of Chronic Subdural Hematoma, Outcome in 1079 Patients
}

\author{
Liang WU, Weiming LIU \\ Capital Medical University, Beijing Tiantan Hospital, Department of Neurosurgery, Beijing, China \\ Corresponding author: Liang WU jasewl@sina.com
}

To the Editor:

W Te read with great interest the recently published article by Mostofi et al. (1), which indicates a minimally invasive surgical technique for the treatment of chronic subdural hematoma. The technique is described as a safe method with favorable outcomes and a good therapeutic alternative to craniostomy. First, we would like to express our appreciation for the authors' effort in this interesting study. However, we have some concerns that we would like to share with the authors.

In Mostofi et al.'s study, patients were evaluated by computed tomography (CT) at 1 week and 6 weeks after surgery. Fortyone $(3.74 \%)$ patients presented a recurrence of hematoma within 6 weeks postoperatively. However, because chronic subdural hematoma usually progresses slowly, a postoperative follow-up of 6 weeks may not be long enough to evaluate the recurrence of hematoma. In the literature, recurrence is generally defined as the occurrence of symptoms attributable to an ipsilateral hematoma seen on a CT scan within 6 months of the original surgical procedure $(2,3)$. Therefore, we suggest that it would be more appropriate if the recurrence rate and clinical outcomes 6 months after surgery can be provided.

\section{REFERENCES}

1. Mostofi K, Peyravi M, Gharaei Moghaddam B: Minimally invasive surgical approach for treatment of chronic subdural hematoma, outcome in 1079 patients. Turk Neurosurg 31(1):18-23, 2021

2. Santarius T, Kirkpatrick PJ, Ganesan D, Chia HL, Jalloh I, Smielewski P, Richards HK, Marcus H, Parker RA, Price SJ, Kirollos RW, Pickard JD, Hutchinson PJ: Use of drains versus no drains after burr-hole evacuation of chronic subdural haematoma: A randomised controlled trial. Lancet 374:10671073, 2009

3. Schucht P, Fischer U, Fung C, Bernasconi C, Fichtner J, Vulcu S, Schöni D, Nowacki A, Wanderer S, Eisenring C, Krähenbühl AK, Mattle HP, Arnold M, Söll N, Tochtermann L, Z'Graggen W, Jünger ST, Gralla J, Mordasini P, Dahlweid FM, Raabe A, Beck J: Follow-up computed tomography after evacuation of chronic subdural hematoma. N Engl J Med 380:1186-1187, 2019 